\title{
Effect of Corona Discharge Treatment on Interface Discharge Behaviour between Polypropylene and Silicone Rubber
}

\author{
Jun-ke GUO*, Chun-yan HAO, Jin-shan YU and Li-qiu LU \\ Tianjin Electric Power Research Institute, State Grid, \\ Tianjin, China \\ Email: hmgaoyu@126.com
}

\begin{abstract}
Electric field distribution in cable joint can be enhanced because its structural complexity, this forms interface discharge between the main insulation of cable and the insulation of cable joint. Polypropylene (PP) has good electrical property and is suitable for being used as insulation material of power cable. On the other hand, silicone rubber (SiR) has been widely used as the insulation of cable joint. The interface between the PP and SiR becomes a weak point where an discharge phenomena may be introduced. In order to improve the electrical property of the interface, a corona discharge treatment has been employed in this research, by which the surface chemical and physical structures of PP can be modified. Obtained results showed that with the treatment of AC corona, the interface breakdown time was obviously extended. It is suggested that the interface breakdown strength can be enhanced through the corona discharge treatment method.
\end{abstract}

Keywords- Polypropylene; Silicone Rubber; Interface Discharge; Corona Discharge Treatment

\section{INTRODUCTION}

Underground electrical power cables have a lot of advantages such as less land occupation, high reliability and so on, they are widely used in power system. It has been pointed out by many researchers that cable joint and terminals are often the weakest point in a cable system. The reason for such a conclusion is that the physical structure of the cable accessory is very complicated. Accordingly, electric field in the accessory is easy to be distorted, resulting in the enhancement of field at some certain points [1]. Therefore, from the viewpoint of safety, it is very important to investigate the interface discharge behavior within a cable joint or a cable terminal.

During the past decades, a lot of articles have been written to clarify the electrical insulation performance within the cable accessory, and it has been noticed that the interface between cable insulation and the accessory often plays a dominant role in its electrical property. It was reported that with the increase of interfacial smoothness, the discharge voltage was increased [2]. It was also revealed that with the increase of the interface pressure, partial discharge could be strengthened [3]. Majid et al. proposed a theory on electric field distribution along the interface and the method of breakdown strength calculation, arguing that the interfacial breakdown strength is governed by the discharge inception stress of the air-filled cavities [4].

Nowadays, PP has been recognized as a promising material for high voltage cable insulation in the future, because of its excellent electrical property and easy to recycle $[5,6]$. However, the interface discharge is still a drawback for the cable joint. Corona discharge can generate active species that vary the surface structures of polymer material, which can improve its surface electrical property. In this paper, isotactic PP and high temperature vulcanized (HTV) SiR were employed as test samples to investigate the effect of AC corona discharge treatment on the interface discharge behavior under $\mathrm{AC}$ voltage. It was found that the interface breakdown strength was improved by means of the $\mathrm{AC}$ corona discharge treatment.

\section{EXPERIMENTAL SETUP}

\section{A. Sample Preparation and Electrode Arrangement}

Isotactic PP (PPH-T03) (Sinopec Beihai company, China) and high temperature vulcanized silicone rubber (110) (Ximei Silicone Materials, China) were employed as test samples. The length and width of the samples were $8 \mathrm{~cm}$ and $5 \mathrm{~cm}$, while the thickness was $5 \mathrm{~mm}$. In order to limit the presence of air between samples, their surfaces were treated as smooth as possible. As shown in Fig. 1, a pair of point to plane electrode system was used for electrode configuration. The PP and SiR samples were kept at a physical pressure of $0.12 \mathrm{MPa}$. The electrodes were formed by attaching $55 \mu \mathrm{m}-$ thick aluminum foil on the surface of i-PP. The point electrode was featured with an angle of $30^{\circ}$. The interval between the point and the plane electrode was $4 \mathrm{~mm}$. Such an electrode arrangement was utilized to simulate the nonuniform electric field distribution at the interface within a real cable joint. 


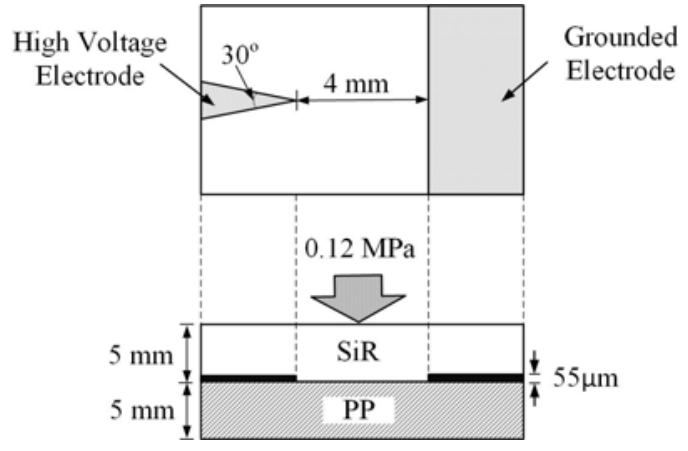

Fig. 1. Schematic diagram of the sample position and the electrode arrangement.

\section{B. Corona Discharge Treatment}

The surface of polypropylene was corona treated by means of the following electrode system in Fig.2. The voltage applied between the needle array and plate electrode was $15 \mathrm{kV}$ with $7.5 \mathrm{kHz}$ of frequency. The distance between the needle tip and the plate electrode was $4 \mathrm{~mm}$. The corona treatment was performed at room temperature with relative humidity of $40 \%$.

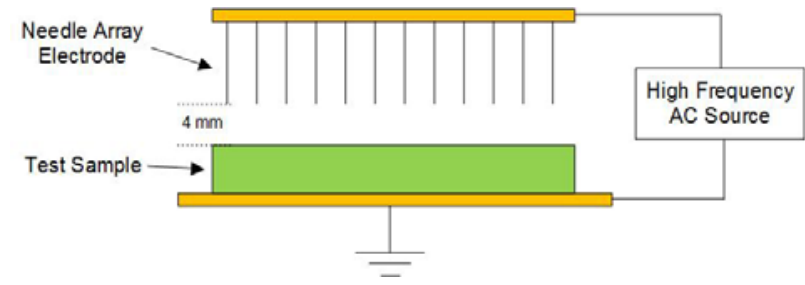

Fig.2 Electrode configuration for corona discharge treatment

\section{Test CIRCUIT AND Procedure}

The test circuit is shown in Fig. 3. A high frequency current transformer (HFCT) was used to measure the discharge current and a high speed $\mathrm{AD}$ convertor was used to collect the data. A charge-coupled device (CCD) camera was used to monitor the propagation of interface discharge channel. Before the test, the sample surface was cleaned with ethyl alcohol and was dried at $40^{\circ} \mathrm{C}$ for 1 hours. The experiment was conducted at room temperature with relative humidity of $45 \%$. By applying AC voltage with $20 \mathrm{kVrms}$ of $50 \mathrm{~Hz}$ between the electrodes, surface discharge was initiated and the discharge current was measured until the insulation interval was breakdown. The PRPD features during the discharge propagation process were extracted and the statistics of discharge magnitude were obtained.

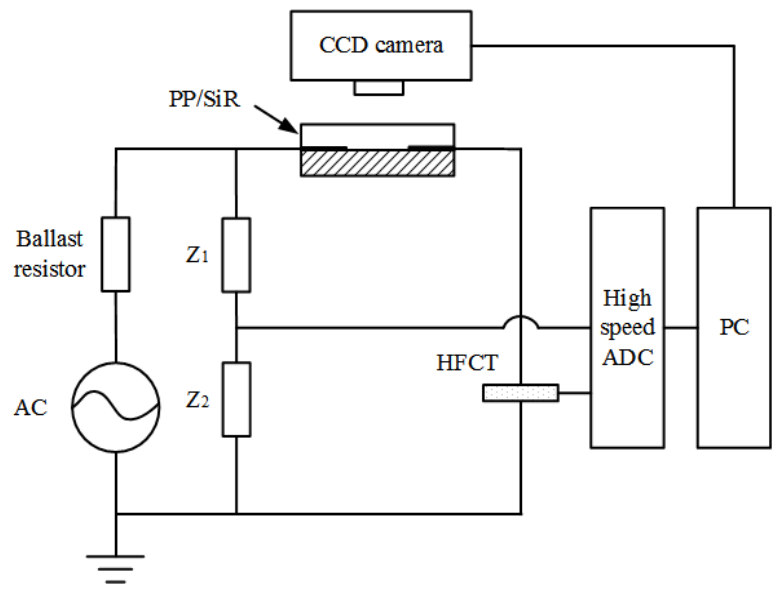

Fig. 3. Schematic diagram of the test circuit.

\section{RESUlTS AND DisCUSSION}

Interface discharge will happen when the high frequency AC voltage was applied between the point to plane electrode. It was found that the average time for the interface breakdown of untreated $\mathrm{PP} / \mathrm{SiR}$ was about $40 \mathrm{~s}$, during such a time period three stages could be found, i.e. (a) discharge initiation, (b) discharge channel propagation and (c) breakdown. The characteristics of the three stages monitored through the CCD camera is shown in Fig.4. Normally, in the stage (a), the discharge pulses are sparse and relatively low. After that, the discharge magnitude and discharge repetition rate increase in stage (b) compared with the former stage (a). Stage (a) lasts for a relatively short time with a typical period of $\sim 15 \mathrm{~s}$, and stage (b) has a time of about $20 \mathrm{~s}$ in general. As regards to the stage (c), the breakdown happens between the electrodes that a discharge arc appears with remarkable light emission and heat, the insulation interval is therefore short-circuited with the permanent loss of insulation property of the interface. Some other features that need to be noticed are those at stage (a) no obvious discharge phenomena could be observed.

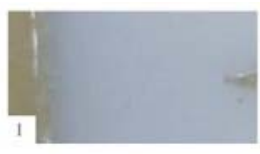

$0-15 s$

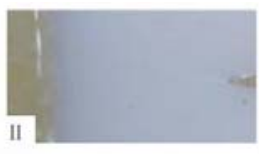

$15-38.1 \mathrm{~s}$

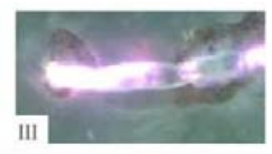

Breakdown
Fig. 4. Interface breakdown phenomena between PP and SiR.

The time to interface breakdown has been employed as an index to estimate the effect of corona discharge treatment on the interfacial electrical strengthe between PP and SiR. It can be observed that an average time to the interfacial breakdown is about $40 \mathrm{~s}$, but with the corona treatment the breakdown time increases to about $80 \mathrm{~s}$, which means that the breakdown strength is enhanced by a factor of 2 . Interface discharge between the PP and SiR has been found an special phenomena which is related to the surface trap distribution as well as surface roughness. At the intial stage, namely stage 
(a), the discharge is more related to surface trap depth. The deeper the surface trap, the more difficuilt the discharge to be induced. At the propagation stage, namely stage(b), the discharge is dependent mainly upon the surface roughness, the larger the roughness is the easier the propagation of discharge channel. By using a mechanical surface roughness measurement equiment, the surface roughness was measured that after the corona discharge treatmen, the roughness decreases from $160 \mathrm{~nm}$ to $80 \mathrm{~nm}$, which indicates that the discharge propagation process is prolonged with the application of corona discharge treatment.

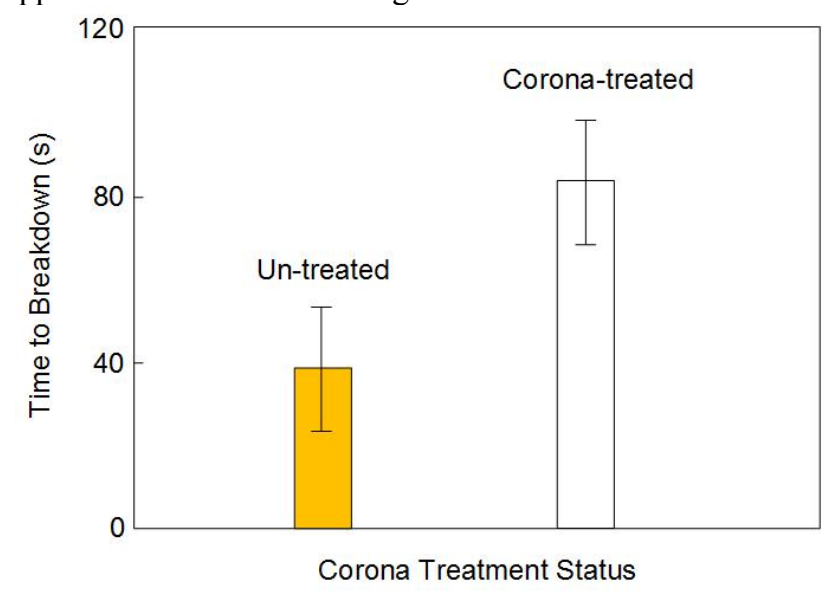

Fig. 5. The time to breakdown for both un-treated and corona discharge treated PP samples.

\section{CONCLUSIONS}

In this paper, the effect of corona discharge treatment on the interface discharge beahviour between PP and SiR has been investigated. The main conclusions are summarized as follows,

1. The discharge process at interface of PP and SiR could be divided into three stages. The first stage is related to the initiation of surface discharge, the second stage is the propagation of surface discharge, and the final stage is the breakdown stage.

2. After the corona discharge treatment, the surface roughness decreases by a factor of 2 , which leads to the extension of breakdown time from $40 \mathrm{~s}$ to $80 \mathrm{~s}$.

Our test results indicate that with the application of corona discharge treatment, the interface electrical strength between PP and SiR can be improved. Such a finding is helpful for the safe operation of PP insulated cable.

\section{REFERENCES}

[1] T. Christen, L. Donzel, and F. Greuter. "Nonlinear resistive electric field grading part 1: Theory and simulation," IEEE Electr. Insul. Mag., vol. 26, no. 6, pp. 47-59, November 2011.

[2] B. X. Du, X. H. Zhu, L. Gu and H. J. Liu. "Effect of surface smoothness on tracking mechanism in XLPE-Si-rubber interfaces," IEEE Trans. Dielectr. Electr. Insul., vol. 18, no. 1, pp. 176-181, February 2011.

[3] L. Zou, Y. Tu, Y. P. Meng, et al. "Effects of interface pressure and surface roughness on the partial discharge between polyethylene and silicone rubber," Insul. Mater., vol. 47, no. 3, pp. 94-102, 2014.

[4] H. Majid and E. Ildstad. "Application of contact analysis on evaluation of breakdown strength and PD inception field strength of solid-solid interfaces," IEEE Trans. Dielectr. Electr. Insul., vol. 19, no. 1, pp. 1-7, February 2012.

[5] S. J. Han, S. Sengupta. Evaluation of rheological and electrical breakdown behaviors of multi-phase polyolefin blends, IEEE Electrical Insulation Conference, Philadelphia, Pennsylvania, USA, pp. 152-156, June 20142014.

[6] C. W. Zhang, Y. D. Huang, S. C. Wang, et al. Blends of Polypropylene and Syndiotactic 1,2-Polybutadiene: Morphology, Crystallization Behaviors and Mechanical Properties, Chemical Research in Chinese Universities, vol. 24, no. 5, pp. 640-643, 2008. 